\title{
Talidomida no tratamento de angiodisplasias intestinais em paciente com síndrome CREST - relato de caso e revisão da literatura
}

\author{
Thalidomide as a treatment of intestinal an- \\ giodisplastic lesions in a patient with CREST \\ syndrome - case report and literature review
}

\author{
Carla Luana Dinardo', Guilherme Henrique Hencklain \\ Fonseca ${ }^{2}$, Sandra Fatima Menosi Gualandro², \\ Paulo Silveira², Liliana Suganuma²
}

Dinardo CL, Fonseca GHH, Gualandro SFM, Silveira P, Suganuma L. Talidomida no tratamento de angiodisplasias intestinais em paciente com síndrome CREST - relato de caso e revisão da literatura. Rev Med (São Paulo). 2009;88(2):103-6.

\begin{abstract}
RESUMO: A anemia ferropriva grave secundária à hemorragia digestiva por angiodisplasias intestinais representa um grande desafio terapêutico. Comumente, as ectasias vasculares são múltiplas e dispersas ao longo do intestino, limitando a eficácia do tratamento hemostático local. Nos últimos anos houve significativo avanço no tratamento anti-angiogênico sistêmico das angiodisplasias intestinais, sendo talidomida a droga mais empregada para tal fim. Relatamos o caso de uma paciente de 49 anos com angiodisplasias intestinais secundárias a síndrome CREST (Calcinose, Raynaud, Dismotilidade Esofágica, Esclerodactilia e Telangiectasias). A paciente apresentava quadro de melena recorrente e alta necessidade transfusional, e não obteve resposta clínica após realização de enteroscopia e eletro-coagulação das lesões com plasma de argônio. Após a introdução de talidomida 100mg ao dia, a paciente evoluiu de forma bastante satisfatória. O caso apresentado neste texto, além de demonstrar sucesso da talidomida no tratamento de angiodisplasias intestinais refratárias à eletro-coagulação com plasma de argônio, também revela eficácia da droga na situação específica da síndrome CREST. Tal fato pode ser de grande valia quando da abordagem de hemorragia intestinal por angiodisplasias nesses pacientes, representando nova opção terapêutica.
\end{abstract}

DESCRITORES: Talidomida. Angiodisplasia. Síndrome CREST. Eletrocoagulação.

1. Médica Residente de Hematologia do Hospital das Clínicas da Faculdade de Medicina da Universidade de São Paulo.

2. Médico(a) Assistente do Departamento de Hematologia do Hospital das Clínicas da Faculdade de Medicina da Universidade de São Paulo.

Endereço para correspondência: Carla Luana Dinardo. Av. Santo Amaro, 5328 Apto. 94. São Paulo, SP. CEP: $04702-000$. E-mail: carlaluana@yahoo.com.br 


\section{INTRODUÇÃO}

A hemorragia digestiva de origem em intestino delgado representa um grande desafio terapêutico. Comumente é decorrente de angiodisplasias localizadas no íleo terminal, fato que dificulta o tratamento hemostático local em virtude do caráter disseminado das lesões.

Nos últimos anos, houve significativo avanço no tratamento anti-angiogênico sistêmico das angiodisplasias intestinais. A droga mais empregada para tal fim é a talidomida, que, além de ação antiinflamatória, apresenta também propriedades anti-angiogênicas, já empregadas para controles de outras doenças, como o mieloma múltiplo. Há relatos de sucesso terapêutico com uso de talidomida em casos de hemorragia digestiva por angiodisplasias esporádicas ou associadas a síndromes clínicas, bem como em casos de sangramento de origem obscura ou associados à doença de Crohn.

Relataremos o caso de uma paciente com angiodisplasia intestinal secundária a esclerodermia forma limitada - síndrome CREST (Calcinose, Raynaud, Dismotilidade Esofágica, Esclerodactilia e Telangiectasias) tratada de forma eficaz com talidomida $100 \mathrm{mg}$ ao dia.

\section{RELATO DE CASO}

Paciente do sexo feminino, 49 anos, foi encaminhada para acompanhamento no Serviço de Hematologia do Hospital das Clínicas da Faculdade de Medicina da Universidade de São Paulo - HCFMUSP em 2003, por anemia ferropriva identificada em exames de rotina.

$\mathrm{Na}$ primeira consulta, foram observados fenômeno de Raynaud, múltiplas telangiectasias em face, esclerodactilia e microstomia. Levantou-se a hipótese de CREST, a qual foi confirmada após detecção dos auto-anticorpos FAN (1/380) e anticentrômero.
Na ocasião, a paciente não apresentava queixas de hipermenorreia, metrorragia ou sangramento intestinal, sendo iniciada reposição de ferro e acompanhamento reumatológico, com introdução de pentoxifilina, prednisona e penicilamina.

Em 2007, quatro anos após diagnóstico de CREST, a paciente começou a apresentar melena, evoluindo com queda significativa de hemoglobina ( $\mathrm{Hb} 5,4 \mathrm{~g} / \mathrm{dL} / \mathrm{Ht}$ 20,4\%) e necessitando de múltiplas internações em serviços de emergência para transfusão de hemocomponentes. Durante internação para investigação do quadro no HCFMUSP, foram realizadas colonoscopia, endoscopia digestiva alta e cápsula endoscópica, que identificaram angiodisplasias localizadas, respectivamente, em reto, em antro gástrico e em múltiplos pontos do intestino delgado, sendo as maiores localizadas na região do jejuno proximal.

Optou-se pela realização de enteroscopia para eletro-coagulação das lesões principais com plasma de argônio. Cerca de uma semana após a alta, a paciente foi novamente internada por persistência de melena e piora da anemia. Realizou-se uma enteroscopia retrógrada, que não visualizou lesões ectásicas após introdução de $1,5 \mathrm{~m}$ do aparelho. Com a persistência do sangramento digestivo e a dificuldade do tratamento endoscópico, a equipe de hematologia introduziu talidomida $100 \mathrm{mg}$ ao dia para controle das angiodisplasias. A talidomida foi administrada apenas após consentimento informado da paciente, com plena orientação sobre os riscos gestacionais e após introdução de método anticoncepcional eficaz.

O sangramento intestinal diminuiu até cessar completamente. Cerca de dois meses após introdução da droga, houve mais um pequeno episódio de sangramento, com remissão espontânea. Atualmente, a paciente completa sete meses de seguimento usando talidomida, sem mais episódios de melena ou qualquer efeito colateral decorrente do uso da droga. A Figura 1 mostra a evolução da hemoglobina da paciente ao longo do acompanhamento.

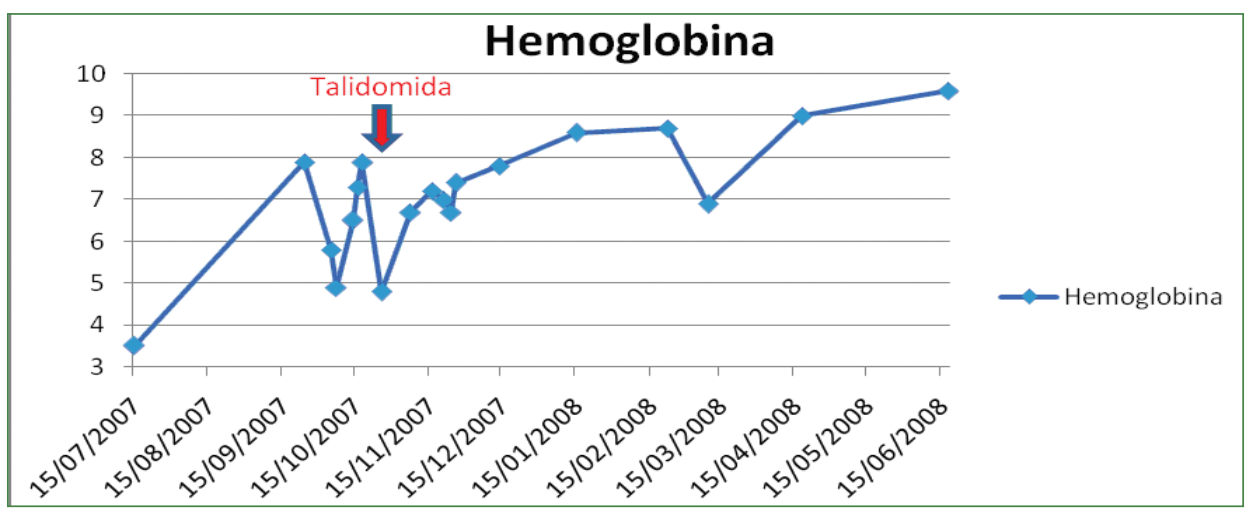

FIGURA 1. Evolução da hemoglobina durante 0 seguimento - Em setembro e outubro de 2007 foram realizadas várias transfusões de hemocomponetes com persistência da anemia. Em novembro de 2007 foi iniciada a talidomida. A partir de então apresentou apenas um episódio de sangramento sem necessidade transfusional. 


\section{DISCUSSÃO}

As angiodisplasias são vasos neoformados presentes na mucosa intestinal que frequentemente causam sangramentos intestinais de repetição. Podem ser esporádicas ou associadas a diversas síndromes, como Klippel-Trenaunay-Weber, EhlersDanlos, CREST e telangiectasia hemorrágica hereditária $^{1,2}$.

Ainda que preferencialmente encontradas no cólon também estão localizadas no intestino delgado (sobretudo íleo terminal), onde representam a principal causa de sangramento. $O$ tratamento de eletro-coagulação com plasma de argônio, apesar de classicamente apontado como de escolha, é desfavorecido pelo fato de as angiodisplasias intestinais serem comumente múltiplas e disseminadas.

Além disso, tanto a realização de várias sessões de eletro-coagulação (visando a abordagem da maior parte das lesões) quanto a realização de procedimentos cirúrgicos intestinais não representa opção terapêutica definitiva para o quadro, visto que lesões angiodisplásicas podem surgir em locais distintos dos inicialmente abordados.

A limitação dos procedimentos acima descritos impulsionou o estudo de opções sistêmicas para tratamento da hemorragia digestiva por angiodisplasias. Dentre as opções estudadas, destacam-se a hormonioterapia combinada com estrogênios e progestágenos, que não se mostrou eficaz no controle de sangramentos em metanálise recentemente publicada, e o octreotide, que em alguns trabalhos mostrou benefício no tratamento das angiodisplasias ${ }^{3-5}$.

As angiodisplasias e outras malformações vasculares são decorrentes de ativação maciça de estágios precoces da angiogênese, com acúmulo de fator de crescimento vascular endotelial (VEGF) e indução de formação de complexos de vasos primitivos que, quando localizados próximos de superfícies mucosas, são susceptíveis a ruptura e sangramento ${ }^{6,7}$.

Em 1994, foi descoberto que a talidomida, droga previamente utilizada como sedativo e antiemético, apresentava ação inibitória sobre VEGF e sobre a angiogênese mediada por fator de crescimento de fibroblasto, além de importante ação anti-inflamatória e imunomoduladora. Acredita-se que tal ação antiangiogênica da talidomida seja a responsável pelos seus efeitos teratogênicos, que culminaram com sua retirada do mercado na década de $60^{\circ}$. Baseado nesta atividade inibitória da angiogênese exibida pela droga iniciou-se sua aplicação na hemorragia digestiva secundária a angiodisplasias, com vários relatos de sucesso clínico ${ }^{9-13}$

Em alguns dos casos relatados, concomitantemente à melhora clínica dos pacientes, houve involução macroscópica das lesões (redução do número, do tamanho e da intensidade da cor), a qual foi identificada em exames com cápsula endoscópica realizados durante o tratamento ${ }^{14}$. Muito provavelmente, a melhora clínica e endoscópica deve-se à ação antiangiogênica da talidomida sobre as angiodisplasias.

Existem relatos de remissão de sangramentos digestivos por mais de dois anos após a suspensão da droga. Outros relatos mostram remissão de sangramento intestinal após uso da talidomida em pacientes com doença de Crohn e em pacientes com sangramentos de causa não identificada ${ }^{11,15}$.

O caso apresentado neste texto, além de demonstrar sucesso da talidomida no tratamento de angiodisplasias intestinais refratárias à eletrocoagulação com plasma de argônio, também revela eficácia da droga na situação específica da esclerose sistêmica forma limitada (síndrome CREST). Tal fato pode ser de grande valia quando da abordagem de hemorragia intestinal por angiodisplasias nesses pacientes, representando nova opção terapêutica.

\section{CONCLUSÃO}

Angiodisplasias de intestino delgado são causa frequente de sangramento digestivo na prática clínica. Relatos de casos sugerem que o uso de talidomida pode levar a remissão clínica dos sangramentos e involução endoscópica das lesões, provavelmente devido à ação antiangiogênica da droga. Relatamos o caso de paciente com angiodisplasias de delgado e CREST, cujo sangramento intestinal cessou após o uso de talidomida $100 \mathrm{mg}$ ao dia. Apesar de faltarem ensaios clínicos controlados para confirmação da eficácia da droga nesta situação, os dados até então publicados permitem concluir que se trata de opção segura e benéfica no tratamento das angiodisplasias intestinais.

Declaração de conflito de interesses Não há conflito de interesses. 
Dinardo CL, Fonseca GHH, Gualandro SFM, Silveira P, Suganuma L. Thalidomide as a treatment of intestinal angiodisplastic lesions in a patient with CREST syndrome - case report and literature review. Rev Med (São Paulo). 2009;88(2):103-6.

\begin{abstract}
The severe ferropenic anemia secondary to digestive bleeding due to intestinal angiodisplastic lesions represents a great challenge. Commonly, angiodisplastic lesions are multiples and disperse through the intestine and that fact limits local treatments. Over the last years, there was a great advance in the antiangiogenic treatment of intestinal angiodisplastic lesions and thalidomide was the most employed drug for this purpose. We report a case of a 49 year-old patient with intestinal angiodisplastic lesions due to CREST syndrome (Calcinosis, Raynaud phenomenon, Esophageal dysmotility, Sclerodactyly, and Telangiectasia). The patient presented repeated episodes of digestive bleeding and did not achieve clinical improvement after enteroscopy and argon plasma coagulation. The treatment consisting of the introduction of thalidomide $100 \mathrm{mg}$ per day demonstrated success. The case presented in this text reveals success in the use of thalidomide in the treatment of intestinal angiodisplastic lesions, probably representing a new therapeutic option.
\end{abstract}

KEY WORDS: Thalidomide. Angiodysplasia. CREST syndrome. Eletrocoagulation.

\title{
REFERÊNCIAS
}

1. Marwick T, Kerlin P. Angiodysplasia of the upper gastrointestinal tract. Clinical spectrum in 41 cases. J Clin Gastroenterol. 1986;8(4):404-7.

2. Dalle I, Geboes K. Vascular lesions of the gastrointestinal tract. Acta Gastroenterol Belg. 2002;65(4):213-9.

3. Junquera F, Saperas E, Videla S, Feu F, Vilaseca J, Armengol JR, et al. Long-term efficacy of octreotide in the prevention of recurrent bleeding from gastrointestinal angiodysplasia. Am J Gastroenterol. 2007;102(2):254-60.

4. Junquera F, Feu F, Papo M, Videla S, Armengol JR, Bordas JM, et al. A multicenter, randomized, clinical trial of hormonal therapy in the prevention of rebleeding from gastrointestinal angiodysplasia. Gastroenterology. 2001;121(5):1073-9.

5. Rossini FP, Arrigoni A, Pennazio M. Octreotide in the treatment of bleeding due to angiodysplasia of the small intestine. Am J Gastroenterol. 1993;88(9):1424-7.

6. Junquera F, Saperas E, de Torres I, Vidal MT, Malagelada JR. Increased expression of angiogenic factors in human colonic angiodysplasia. Am J Gastroenterol. 1999;94(4):1070-6.

7. Bauditz J, Lochs H. Angiogenesis and vascular malformations: antiangiogenic drugs for treatment of gastrointestinal bleeding. World J Gastroenterol. 2007;13(45):5979-84.

8. Joussen AM, Germann T, Kirchhof B. Effect of thalidomide and structurally related compounds on corneal angiogenesis is comparable to their teratological potency. Graefes Arch Clin Exp Ophthalmol. 1999;237(12):952-61.

9. Shurafa M, Kamboj G. Thalidomide for the treatment of bleeding angiodysplasias. Am J Gastroenterol. 2003;98(1):221-2.

10. Heidt J, Langers AM, van der Meer FJ, Brouwer RE. Thalidomide as treatment for digestive tract angiodysplasias. Neth J Med. 2006;64(11):425-8.

11. Bauditz J, Schachschal G, Wedel S, Lochs H. Thalidomide for treatment of severe intestinal bleeding. Gut. 2004;53(4):609-12.

12. de Koning DB, Drenth JP, Friederich $P$, Nagengast FM. Thalidomide for the treatment of recurrent gastrointestinal blood loss due to intestinal angiodysplasias. Ned Tijdschr Geneeskd. 2006;150(36):1994-7.

13. Hirri HM, Green PJ, Lindsay J. Von Willebrand's disease and angiodysplasia treated with thalidomide. Haemophilia. 2006;12(3):285-6.

14. Bauditz J, Lochs H, Voderholzer W. Macroscopic appearance of intestinal angiodysplasias under antiangiogenic treatment with thalidomide. Endoscopy. 2006;38(10):1036-9.

15. Wettstein AR, Meagher AP. Thalidomide in Crohn's disease. Lancet. 1997;350(9089):1445-6. 